\title{
Manejo preoperatorio de medicamentos en pacientes hipertensos*
}

\author{
Dr. CLAUDIO NAZAR J. ${ }^{1}$, Int. CHRISTIAN HERRERA F. ${ }^{2}$, ALEJANDRO GONZÁLEZ A. ${ }^{1}$
}

1 División de Anestesiología.

2 Interno de Medicina.

Facultad de Medicina, Pontificia Universidad Católica de Chile.

Santiago, Chile.

\begin{abstract}
Preoperative pharmacological management of hypertensive patients

The proportion of older hypertensive patients that require a surgical procedure has increased in the last years. These patients require a through preoperative assessment, considering the medications in use, laboratory and images. An adequate pharmacological management of patients with hypertension in the perioperative period will prevent cardiovascular complications. Therefore the health care team must assure that patients with hypertension will be operated in optimal conditions.
\end{abstract}

Key words: Hypertension, preoperative assessment, medications.

\section{Resumen}

La hipertensión arterial es una enfermedad con alta prevalencia en la población chilena, llegando a casi el 75\% en el grupo de mayores de 65 años. En el ámbito quirúrgico, el número de pacientes de edad mayor y que padecen hipertensión arterial ha aumentado significativamente, lo que nos obliga a realizar una adecuada y detallada evaluación preoperatoria del paciente hipertenso con el fin de conocer su condición al momento de la cirugía, los medicamentos antihipertensivos que utiliza y solicitar los exámenes de laboratorio y/o de imágenes necesarios. Una adecuada asesoría al paciente respecto al manejo de su medicación antihipertensiva preoperatoria pretende disminuir las complicaciones en todo el período perioperatorio, tanto por su suspensión como por su mantención. De esta forma se busca establecer las medidas que permitan al paciente enfrentar el procedimiento quirúrgico en las mejores condiciones posibles.

Palabras clave: Hipertensión arterial, manejo preoperatorio, medicamentos antihipertensivos.

*Recibido el 8 de noviembre de 2012 y aceptado para publicación el 12 de enero de 2013.

Los autores no refieren conflictos de interés.

Correspondencia: Dr. Claudio Nazar J.

Marcoleta 367, 3er piso, Santiago, Chile. Fax: 6327620. Código postal: 6510260.

cenazar@med.puc.cl 


\section{Introducción}

Con el aumento en la expectativa de vida de la población, asociado al perfeccionamiento de las técnicas quirúrgicas, es más frecuente ver pacientes de mayor edad siendo sometidos a cirugías de creciente complejidad ${ }^{1}$. Junto con esto se ha observado un aumento en la prevalencia de patologías no relacionadas a la causa de la cirugía en estos pacientes, así como también el uso de terapia farmacológica para el control de dichas comorbilidades ${ }^{2}$.

Dentro de las comorbilidades más frecuentes en la población chilena se encuentra la hipertensión arterial, que según la Encuesta Nacional de Salud 2009 , está presente en el $26,9 \%$ de la población ${ }^{3}$. $\mathrm{Si}$ se analiza por grupo etario, en el rango de 45-64 años la prevalencia de hipertensión alcanza el 43,8\% de la población, mientras que en mayores de 65 años llega al $74,6 \%$.

No se encontraron datos locales respecto a la prevalencia de esta condición en pacientes quirúrgicos, sin embargo, Errando y $\operatorname{cols}^{4}$, hacen referencia que la prevalencia poblacional es extrapolable al grupo de pacientes que se someten a una intervención quirúrgica. Datos obtenidos en nuestra institución, provenientes del registro computacional de la visita preanestésica de 28.810 pacientes, muestran una prevalencia de hipertensión moderada y severa de $18,6 \%$, lo que es menor a lo informado en la Encuesta Nacional de Salud. Esta diferencia puede ser explicada por desconocimiento del paciente de su condición de hipertenso o por subregistro en el sistema computacional. Según un estudio de Kennedy y $\operatorname{cols}^{5}$, el $50 \%$ de los pacientes quirúrgicos consume al menos un medicamento antihipertensivo al momento de indicarse la realización de una cirugía ${ }^{5}$.

El consumo de medicamentos en el período preoperatorio es un tema importante dentro del contexto quirúrgico. Se ha encontrado un aumento de hasta 3 veces en las complicaciones quirúrgicas en la población que consume algún medicamento antes de la cirugía en comparación a la población que no utiliza fármacos $^{5,6}$. Dentro de las complicaciones postoperatorias que sufren los pacientes que consumen medicamentos, un $5 \%$ es atribuible directamente a la suspensión de éste 5 .

En la literatura se encuentran guías que buscan orientar al clínico en el manejo de la medicación crónica de los pacientes en el preoperatorio, con bajo nivel de evidencia científica, la gran mayoría fundamentada en la opinión de expertos. En nuestro país no existen guías clínicas al respecto y las indicaciones de qué medicamentos suspender y el momento de hacerlo se basa en la experiencia del clínico, por lo que es posible observar distintas tendencias entre los médicos que se ven enfrentados al manejo preoperatorio de un paciente quirúrgico.

Esta revisión pretende realizar una actualización en el manejo de la medicación preoperatoria más usada por los pacientes hipertensos que serán sometidos a cirugía electiva y esbozar recomendaciones de continuación o suspensión para cada uno de ellos.

\section{Análisis por tipo de medicamento antihipertensivo}

\section{Inhibidores de enzima convertidora de angiotensina-antagonista receptor angiotensina II (iECA-ARA II)}

Los iECA y ARA II son los medicamentos más usados en pacientes hipertensos y con efectividad ampliamente comprobada, sobre todo en la población de pacientes con factores de riesgo cardiovascular y enfermedad coronaria ${ }^{7}$.

Se encontraron trabajos que intentan orientar su uso en el período preoperatorio, pero insisten en que el tema continúa siendo controversial y se basan en opinión de expertos para entregar sus recomendaciones.

Como efecto negativo de sus usos, ambas drogas se han asociado a la aparición de hipotensión en los 30 min siguientes a la inducción anestésica, lo que hace necesario la utilización de drogas vasopresoras para restablecer la hemodinamia (efedrina 4-8 mg endovenoso, fenilefrina 25-50 ug endovenoso), siendo en algunas ocasiones refractarias al uso de drogas en dosis habituales, requiriendo aumentar las dosis para controlar la hipotensión con los consiguientes riesgos que esto conlleva ${ }^{8}$.

Estudios recientes ${ }^{9}$, relacionan el desarrollo de estos episodios de hipotensión con aumento en la morbilidad postoperatoria, frecuentemente falla renal e incluso la muerte, lo que contrasta con lo encontrado por Miceli y cols ${ }^{10}$, quienes no lograron demostrar que existiera relación entre la hipotensión post inducción anestésica y el aumento de la mortalidad. Además, Colson y cols ${ }^{11}$ han mostrado que en pacientes que continuaron usando iECA/ARA II en el preoperatorio se podría ver una mejoría en la función renal, planteando un rol de protección de esta última.

Dentro de los tipos de cirugías en los cuales se ha estudiado el efecto del uso de iECA en el período preoperatorio se encuentran las intervenciones cardiovasculares. Ouzounian y cols ${ }^{12}$, evaluaron el impacto de estas drogas en pacientes que fueron sometidos a cirugía de by pass coronario, concluyendo que no existía relación entre la terapia preoperatoria con iECA y resultados adversos postquirúrgicos ${ }^{12}$, comportándose más bien como factor no relacionado. 
Comfere y cols ${ }^{7}$, compararon los efectos de la suspensión versus la mantención de iECA y ARA II en el preoperatorio, encontrando un aumento en el número de episodios de hipertensión postoperatoria de difícil manejo en el grupo de pacientes que suspendió la medicación el día previo a la cirugía. Por su parte, Bertrand y cols ${ }^{13}$, concluyeron que en el período postoperatorio el desarrollo de hipertensión fue igual en ambos grupos de pacientes, lo cual viene a confirmar la incertidumbre respecto a la conducta a seguir con esta familia de drogas en el período preoperatorio.

Castanheira y $\operatorname{cols}^{1}$, en base a una revisión sistemática y en consenso de expertos (encuesta), recomiendan suspender los iECA y ARA II $24 \mathrm{~h}$ antes de la cirugía. Aún así, se sugiere evaluar la indicación en cada paciente, ya que ciertos casos, como hipertensión no controlada o insuficiencia cardíaca con disfunción sistólica, se beneficiarían de la continuación de iECA/ARA II, pero teniendo precaución en mantener una monitorización intraoperatoria y volemización adecuadas antes de la inducción anestésica e inicio de la cirugía?

\section{Beta bloqueadores}

Existía una tendencia a suspender los medicamentos beta bloqueadores previo a la cirugía argumentando que su acción cronótropa negativa aumentaba el riesgo de bradicardia e hipotensión ${ }^{14}$. Actualmente, estudios han evaluado el uso de beta bloqueadores en pacientes que han sido sometidos a cirugía vascular, encontrando que la suspensión de éstos se asocia a un aumento en el riesgo de desarrollar isquemia miocárdica con un impacto en la morbilidad y mortalidad post operatoria ${ }^{15}$. En esa misma línea de investigación, Broecke y cols ${ }^{16}$, encontraron una reducción en la mortalidad a 30 días en pacientes sometidos a cirugía cardíaca. Se postula que al disminuir el tono simpático, y de esta forma la respuesta cardíaca al estímulo quirúrgico, se reducen los requerimientos de oxígeno miocárdico, contribuyendo incluso a controlar el desarrollo de arritmias durante la intervención quirúrgica. Además, disminuirían las respuestas reflejas a la hipovolemia, por lo que se requiere de monitorización del estado hídrico del paciente en el período perioperatorio.

Debido a los efectos positivos que presentan los beta bloqueadores y a que la suspensión de ellos en el período preoperatorio, sobre todo en usuarios de forma crónica, se asocia a aumento en desarrollo de isquemia, morbilidad y mortalidad ${ }^{14}$, se recomienda la mantención de estos medicamentos antes de la cirugía y su continuación posterior apenas sea posible.

\section{Bloqueadores canales de calcio}

En la literatura médica no existen estudios comparativos entre la suspensión y continuación de blo- queadores de canales de calcio en el preoperatorio. La limitada evidencia muestra asociación entre el uso de estos medicamentos y reducción de la mortalidad en el período post operatorio ${ }^{1}$. Este efecto se ha atribuido a que los bloqueadores de canales de calcio entregan mayor estabilidad hemodinámica, reducen la isquemia y las arritmias en el paciente quirúrgico ${ }^{17}$. Se ha encontrado también un aumento en el riesgo de sangrado en pacientes que usan bloqueadores de canales de calcio en forma crónica, pero estos datos han sido contradictorios ${ }^{14}$.

No se ha descrito presencia de complicaciones mayores debido a la suspensión de bloqueadores de canales de calcio.

A pesar de la escasa evidencia existente, se recomienda continuar el uso de bloqueadores de canales de calcio antes del procedimiento quirúrgico, dado el probable beneficio en disminuir la mortalidad post operatoria ${ }^{1,14,17}$.

\section{Diuréticos}

Tampoco existen ensayos comparativos respecto al uso o descontinuación de diuréticos (tiazidas y de asa) en el período preoperatorio. Kheterpal y $\operatorname{cols}^{18}$, han mostrado mayor riesgo a presentar hipotensión en aquellos pacientes que se encuentran en tratamiento con diuréticos asociados a iECA/ARA II, mismo efecto encontrado en paciente que sólo utilizan iECA/ARA II.

Debido a su mecanismo de acción, se han encontrado alteraciones hidroelectrolíticas, entre ellas de la kalemia y natremia, por lo que el clínico debe estar atento a los niveles séricos de potasio y sodio en el preoperatorio, así como en la volemia del paciente ${ }^{14}$. Además, los diuréticos son la principal causa de hiponatremia en los pacientes adultos ${ }^{19}$, por lo que en la evaluación preoperatoria se debe buscar activamente a los pacientes con esta condición.

La recomendación sugiere descontinuar los diuréticos $24 \mathrm{~h}$ antes o en la mañana de la cirugía, reiniciándolos una vez restablecida la ingesta oral de líquidos por parte del paciente ${ }^{1,2}$.

\section{Conclusiones}

La hipertensión arterial es un problema de salud de importancia en la población general, como también en los pacientes que son sometidos a cirugía, lo que ha aumentado en la última época junto con el mejoramiento de las técnicas quirúrgicas que permiten cirugías en los pacientes de edad mayor. Esto también ha significado adecuar el manejo de los fármacos antihipertensivos en el período preoperatorio para optimizar la condición del paciente que será sometido a cirugía, dado que se han de- 
mostrado complicaciones asociadas tanto al uso de estos fármacos como a su suspensión. Dentro de las drogas más usadas se encuentran los iECA y ARA II, los cuales se han asociado a un incremento de los episodios de hipotensión moderada-severa posterior a inducción anestésica, los cuales son manejados por el especialista con volumen y drogas vasoactivas endovenosas. Los beta bloqueadores se pueden continuar administrando dado el beneficio demostrado en disminuir el riesgo de isquemia miocárdica postoperatoria. Para los bloqueadores de canales de calcio existe escasa literatura, pero se recomienda continuar debido al eventual efecto benéfico. Finalmente, los diuréticos también se han relacionado a episodios de hipotensión y alteraciones electrolíticas, por lo que se recomienda suspender $24 \mathrm{~h}$ antes o la misma mañana de la cirugía.

En nuestra institución, recomendamos a los pacientes consumir sus fármacos antihipertensivos con un pequeño sorbo de agua, si éstos están indicados el mismo día de la cirugía, para mantener las condiciones de ayuno previo a esta, aún cuando se carecen de estudios clínicos que avalen esta conducta.

Se hace necesario el desarrollo de más estudios comparativos que evalúen los efectos de la continuación o suspensión de los medicamentos antihipertensivos, para que sea posible establecer guías clínicas que orienten el manejo de fármacos en el período preoperatorio con el fin de mejorar la seguridad de las intervenciones quirúrgicas para los pacientes y terminar así con las controversias existentes.

\section{Referencias}

1. Castanheira L, Fresco P, Macedo A. Guidelines for the management of chronic medication in the perioperative period: systematic review and formal consensus. J Clin Pharm Ther. 2011;36:446-67.

2. Juvany R, Mercadal G, Jodar R. Manejo perioperatorio de la medicación crónica no relacionada con la cirugía. An Med Interna 2004;21:291-300.

3. Ministerio de Salud Gobierno de Chile, Facultad de Medicina Pontificia Universidad Católica de Chile, Observatorio Social Universidad Alberto Hurtado. Encuesta nacional de salud 2009-2010 Tomo II, V. Resultados, Presión Arterial:13-35.

4. Errando CL, Vila M, Armário P. Hipertensión Arterial y el paciente quirúrgico. Rev Esp Anestesiol Reanim. 2009;56:465-6.

5. Kennedy JM, van Rij AM, Spears GF, Pettigrew RA, Tucker IG. Polypharmacy in a general surgical unit and consequences of drug withdrawal. Br J Clin Pharmacol. 2000;49:353-62.
6. Noble DW, Kehlet H. Risks of interrupting drug treatment before surgery. BMJ 2000;321:719-20.

7. Comfere T, Sprung J, Kumar M, Draper M, Wilson D, Williams B, et al. Angiotensin system inhibitors in a general surgical population. Anesth Analg. 2005;100:63644.

8. Ashraf W, Wong D, Ronayne M, Williams D. Guidelines for preoperative administration of patients' home medications. J Perianesth Nurs. 2004;19:228-33.

9. Auron M, Harte B, Kumar A, Michota F. Reninangiotensin system antagonists in the perioperative setting: clinical consequences and recommendations for practice. Postgrad Med J. 2011;87:472-81.

10. Miceli A, Capoun R, Fino C, Narayan P, Bryan AJ, Angelini GD, et al. Effects of angiotensin-converting enzyme inhibitor therapy on clinical outcome in patients undergoing coronary artery bypass grafting. J Am Coll Cardiol. 2009;54:1778-84.

11. Colson P, Ribstein J, Mimran A, Grolleau D, Chaptal PA, Roquefeuil B. Effect of angiotensin converting enzyme inhibition on blood pressure and renal function during open heart surgery. Anesthesiology 1990;72:237.

12. Ouzounian M, Buth KH, Valeeva L , Morton CC, Hassan A, Ali IS. Impact of preoperative angiotensinconverting enzyme inhibitor use on clinical outcomes after cardiac surgery. Ann Thorac Surg. 2012;93:55964.

13. Bertrand M, Godet G, Meersschaert K, Brun L, Salcedo E, Coriat P. Should the angiotensin II antagonists be discontinued before surgery? Anesth Analg. 2001;92:26-30.

14. Muluk V, Macpherson D. Perioperative medication management. UpToDate Inc. Disponible en www.uptodate. com (consultado el 23 de abril de 2012).

15. Shammash J, Trost J, Gold J, Berlin J, Golden M, Kimmel S. Perioperative beta-blocker withdrawal and mortality in vascular surgical patients. Am Heart J. 2001;141:148-53.

16. Ten Broecke P, De Hert S, Mertens E, Adriaensen $H$. Effect of preoperative beta-blockade on perioperative mortality in coronary surgery. $\mathrm{Br} \mathrm{J}$ Anaesth. 2003;90:27-31.

17. Wijeysundera D, BeattieW. Calcium channel blockers for reducing cardiac morbidity after noncardiacsurgery: a meta-analysis. Anesth Analg. 2003;97:634-41 .

18. Kheterpal S, Khodaparast O, Shanks A, O’Reilly M, Tremper K. Chronic angiotensin-converting enzyme inhibitor or angiotensin receptor blocker therapy combined with diuretic therapy is associated with increased episodes of hypotension in noncardiac surgery. J Cardiothorac Vasc Anesth. 2008; 22:180-6.

19. Androgué H, Madias N. Hyponatremia. N Engl J Med. 200;342:1581-9. 